\title{
Azygos vein cannulation: recognition is vital for preventing complications
}

\author{
Ragesh Panikkath MD, Sian Yik Lim MD, Deepa Panikkath MD
}

\begin{abstract}
Inadvertent cannulation of the azygos vein can occur during central vein cannulations, especially from the left side. This can cause several complications, including rupture of the azygos vein. This complication is unlikely from the more commonly used right internal jugular vein access, although that approach is not free of complications. An abrupt curve at the tip of the central venous catheter showing venous wave forms and high oxygen saturations suggest azygos vein cannulation. Azygos vein cannulations may be more common in patients with heart failure in which the vein is dilated.
\end{abstract}

Key words: Azygos vein, central venous line, central venous catheter, cannulation

\section{INTRODUCTION}

Cannulation of the central veins is an essential technique for both long term and emergent care since these catheters allow high volume fluid resuscitation, administration of colloids, vasopressors, and blood, and hemodynamic monitoring. Although generally safe, especially with use of ultrasound guidance, these catheters can cause significant complications which can be lethal, if not recognized. Possible complications include rupture of the internal carotid artery, pneumothorax, rupture of the vein, thrombosis, obstruction of the airway, and infection. Azygos vein cannulation is a rare complication of central venous access $(1.2 \%)$, especially with access from the right side. We present an unusual case of displacement of the right subclavian catheter tip into the azygos vein during CPR.

Corresponding author: Ragesh Panikkath MD Contact Information: ragesh.panikkath@ttuhsc.edu DOI: $10.12746 /$ swrccc2013.0104.045

\section{Case Presenttion}

A 72-year-old gentleman with history of coronary artery disease (status post coronary artery bypass surgery) and defibrillator implantation for severe left ventricular systolic dysfunction presented to the emergency department (ED) after a resuscitated outof-hospital cardiac arrest. He arrested twice again in the ED. A right subclavian triple lumen central venous catheter (CVC) was inserted for medication delivery. During insertion, dark blood with minimal back bleed suggested venous access. The waveforms also suggested a venous position. However, a blood gas from the distal port of CVC showed an oxygen saturation of $94 \%$. Connection of the CVC to a pressure transducer revealed venous wave forms with a pressure of 7 $\mathrm{mmHg}$. The CVC was noted to be in the superior vena cava with the tip turned medially in the chest $x$-ray (Figure 1). Although return of spontaneous circulation could be attained transiently, the patient had cardiac arrest multiple times and could not be resuscitated. 


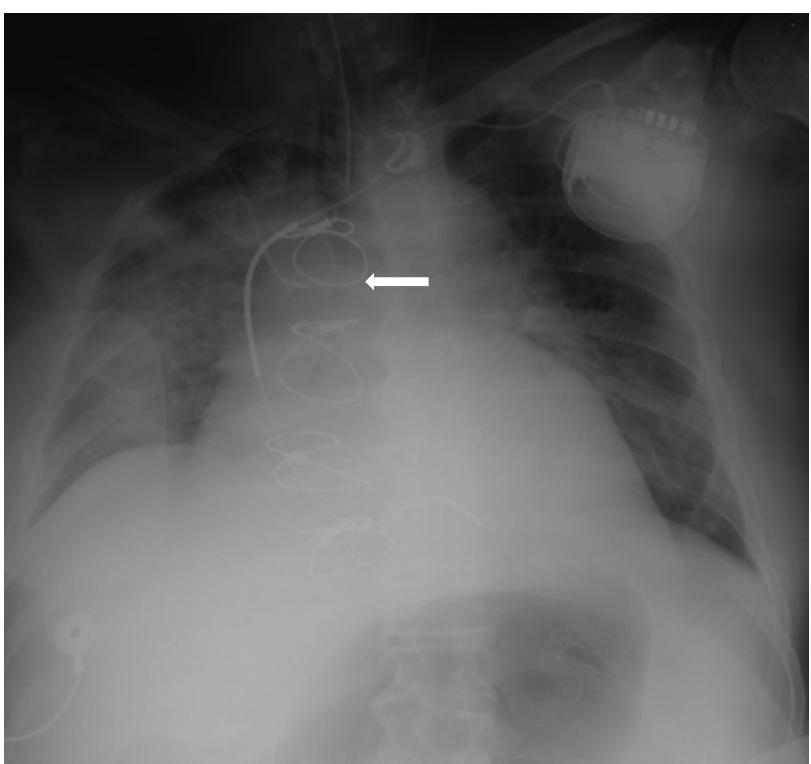

Figure 1. Anteroposterior chest radiograph showing the central venous line in the superior vena cava with its tip in the azygos vein (pointed by white arrow)

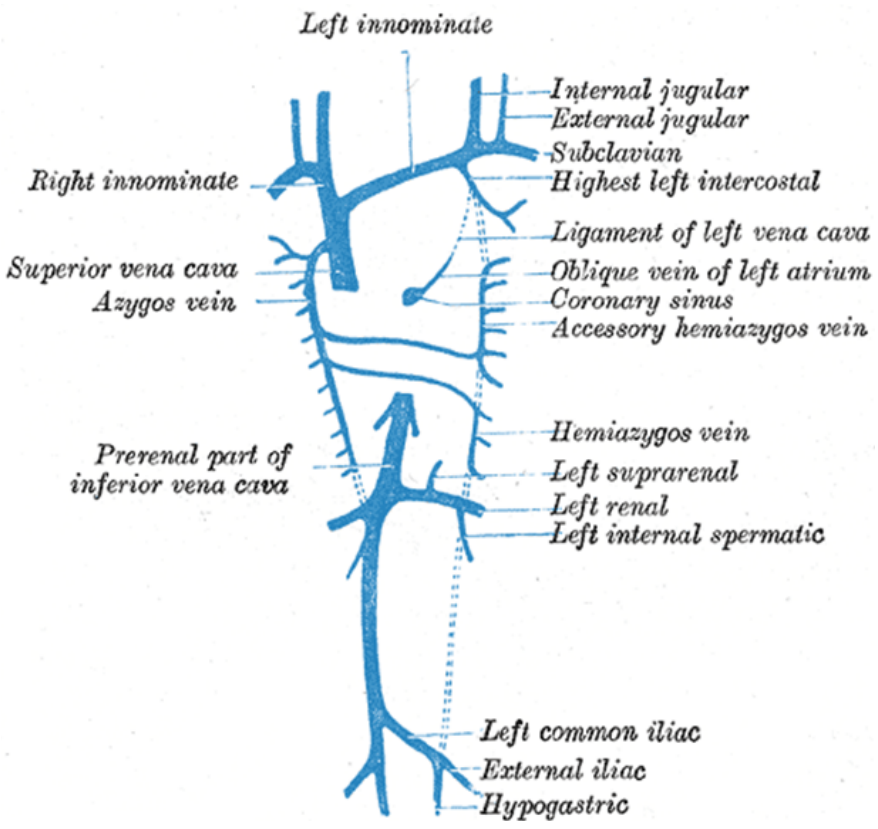

Figure 2. Diagram of azygos vein and other major veins in the body (image courtesy Wikipedia commons; http://en.wikipedia.org/wiki/File:Gray480.png; accessed 08/28/2013)

\section{Discussion}

The curvature at the tip and the high oxygen saturations in a location showing venous wave forms can be explained by inadvertent azygos vein cannulation by the CVC tip. The bend at the tip of the CVC points to the location of CVC tip in the azygos vein. The oxygen saturation from azygos vein can be as high as $92 \%$ on room air and is usually higher than that of the superior vena cava. ${ }^{2}$ Significant variation exists in azygos vein anatomy, with renal veins draining into it being one of the explanations for high oxygen saturations. ${ }^{2}$ A differential diagnosis for this situation is inadvertent arterial cannulation. However, this would have shown the CVC crossing the midline and following a path leading to the arch of the aorta. Inadvertent azygos cannulation more frequently occurs in conditions in which it might be dilated (as in heart failure) and needs to be recognized since it carries a risk of rupture of the vessel.

Central veins provide a good access for volume resuscitation and vasopressors in patients who are critically ill. However, complications can arise from the common procedure which may be lethal if not recognized in a timely manner. In a study of 1,287 post procedural radiologic examinations of central venous cannulations, CVC malposition in the azygos vein was found in $16(1.2 \%))^{3}$ Eleven $(69 \%)$ of these catheters had been inserted through the left subclavian vein, three $(19 \%)$ in the left jugular vein, two $(12 \%)$ in the right subclavian vein, and none $(0 \%)$ in the right jugular vein. It was reported in this study that there was a statistically significant difference in the frequency of azygos arch cannulation between left- and right-sided catheters $(p=0.001)$. The only complication in this study was perforation of the azygos which was seen in three of 16 cases (19\%). Malposition in the azygos vein is extremely rare from the right internal jugular vein with only one case report of this situation. However, this patient had rupture of the azygos vein with right hemothorax requiring video assisted surgery for repair.

In anteroposterior chest x-rays, the curvature at the tip of the central line or "down the barrel" ap- 
pearance (end on appearance of a curved tip of the central line) points to the location in the azygos vein. Haygood et al compared 30 cases of azygos venous placement and 30 cases of superior vena cava placement and suggested that if the tip of the central line extends at least $15 \mathrm{~mm}$ caudal to the cephalic edge of the right main bronchus without any "down-the-barrel" or curved caudal appearance, it can be classified as in the SVC in nearly $100 \%$ of cases. They suggest that in such cases lateral chest $\mathrm{x}$-ray can be safely avoided, reducing cost and radiation exposure. ${ }^{1}$ This is important in critically ill patients in intensive care units, where lateral chest radiographs are often not feasible. Although considered a catheter malposition with the risk of complications during central venous catheter placement, placement of defibrillator leads in the azygos vein has been found to offer a lower defibrillation threshold (DFT) in patients with high DFTs. No complications have been reported with this approach. $^{4,5}$

The azygos vein develops from the fetal right posterior cardinal and supracardinal veins. An azygos (unpaired) (Greek zyg=paired) vein is an unpaired vein lying on the right side of the vertebral column draining blood from the posterior thorax and abdomen into the superior vena cava. It forms an important channel connecting the superior and inferior vena cava in cases of obstruction of either. The anatomy of this vessel can be variable, and it can drain blood from the thoracic veins, bronchial veins, and even gonadal veins. The azygos vein forms opposite the first and second lumbar vertebrae; it later ascends into the thorax through the aortic hiatus of diaphragm to ascend in the posterior mediastinum and arches over the right main bronchus at the root of the right lung to join the superior vena cava. Entry of the CVC into the arch of the azygos creates the abrupt bend described above.

\section{KEYPOINTS}

1. An abrupt bend at the tip of a central venous catheter and high oxygen saturations point to its probable location in the azygos vein.
2. This can cause significant complications, incluidng rupture of the vein.

Author Affiliation: Ragesh Panikkath, Sian Yik Lim, and Deepa Panikkath are residents in Internal Medicine at TTUHSC, Lubbock, TX.

Received: 08/13/2013

Accepted: 08/28/2013

Reviewers: Michael Phy DO, Kenneth Nugent MD

Published electronically: 10/15/2013

Conflict of Interest Disclosures: None

\section{REFERENCES}

1. Haygood TM, Brennan PC, Ryan J, Yamal JM, Liles L, O'Sullivan P, Costelloe CM, Fitzgerald NE, Murphy WA, Jr. Central venous line placement in the superior vena cava and the azygosgos vein: differentiation on posteroanterior chest radiographs. AJR Am J Roentgenol 2011;196:783-7.

2. Jain KK, Wagner HR, Lambert EC. Comparison of oxygen saturation of blood in azygosgos vein and superior vena cava. Circulation 1970;41:55-8.

3. Bankier AA, Mallek R, Wiesmayr MN, Fleischmann D, Kranz A, Kontrus M, Knapp S, Winkelbauer FW. Azygosgos arch cannulation by central venous catheters: radiographic detection of malposition and subsequent complications. J Thorac Imaging 1997;12:64-9. 
4. Cooper JA, Latacha MP, Soto GE, Garmany RG, Gleva MJ, Chen J, Faddis MN, Smith TW. The azygosgos defibrillator lead for elevated defibrillation thresholds: implant technique, lead stability, and patient series. Pacing Clin Electrophysiol 2008;31:1405-10.

5. Cooper JA, Smith TW. How to implant a defibrillation coil in the azygosgous vein. Heart Rhythm 2009;6:1677-80. 